\title{
Nontuberculous mycobacterial disease and Aspergillus-related lung disease in bronchiectasis
}

\author{
H. Kunst*, M. Wickremasinghe ${ }^{\#}$, A. Wells ${ }^{\#}$ and R. Wilson ${ }^{\#}$
}

ABSTRACT: The aim of the present study was to determine whether patients with bronchiectasis and nontuberculous mycobacteria (NTM) have a higher prevalence of Aspergillus-related lung disease.

A series of 30 consecutive patients with bronchiectasis and NTM (cases) were compared with 61 patients with bronchiectasis and no evidence of NTM (controls). Aspergillus serology and computerised tomography of the thorax were used to identify Aspergillus-related lung diseases, including aspergilloma, allergic bronchopulmonary aspergillosis and chronic necrotising pulmonary aspergillosis.

The rate of positive Aspergillus serology was higher in cases with NTM disease compared with controls (10 out of 30 versus six out of 61 ). The radiological features of Aspergillus-related lung disease were also more common among patients with NTM disease than controls (six out of $\mathbf{3 0}$ versus none out of 61). This association between NTM disease and Aspergillus-related lung disease remained significant after adjustment for confounding effects of age and lung function (adjusted odds ratio 5.1, 95\% confidence interval 1.5-17.0).

Patients with bronchiectasis and nontuberculous mycobacterial disease have a higher prevalence of coexisting Aspergillus-related lung disease than patients with bronchiectasis and without nontuberculous mycobacteria. Identification of Aspergillus-related lung disease is important as prognosis amongst undetected cases is invariably poor.

KEYWORDS: Allergic bronchopulmonary aspergillosis, aspergilloma, Aspergillus, bronchiectasis, chronic necrotising pulmonary aspergillosis, nontuberculous mycobacteria

$\mathbf{N}$ ontuberculous mycobacteria (NTM) pulmonary disease, particularly that due to Mycobacterium xenopi, M. kansasii and $M$. malmoense, has increased in recent years [1, 2]. Patients infected with NTM may have coexisting lung disease, such as bronchiectasis or chronic obstructive pulmonary disease, which can interfere with accurate differential diagnosis at presentation and with assessment of response to subsequent treatment. Patients with chronic lung disease are also prone to Aspergillus colonisation and infection [3]. Cystic fibrosis (CF) and NTM infection may be associated with allergic bronchopulmonary aspergillosis (ABPA) and steroid therapy [4].

Diagnosis of Aspergillus-related lung disease is based on tests such as serology, respiratory specimen (sputum or bronchoalveolar lavage) examination and radiology, but none of these is a satisfactory gold standard. In addition, sputum examination for Aspergillus fumigatus is often unreliable $[5,6]$. Pulmonary disease caused by A. fumigatus is traditionally classified into three distinct groups, namely aspergilloma, ABPA and invasive aspergillosis. There may be a clinical and radiological overlap between these groups as more than one Aspergillus-related lung disease can coexist [7]. Invasive aspergillosis has been further categorised as angio-invasive and acute or chronic airway invasive aspergillosis (chronic necrotising pulmonary aspergillosis (CNPA) or semi-invasive aspergillosis) [7,8]. The former is an aggressive form of aspergillosis seen in severe immunosuppression, whereas the latter is an indolent form of invasive aspergillosis, which has been associated with NTM infection [9].

NTM disease itself may prove difficult to diagnose due to its nonspecific clinical and radiographical presentation, although characteristic radiographical features of some NTM species have been described [10-12]. NTM infection and concomitant Aspergillus-related lung disease

\section{AFFILIATIONS}

${ }^{*}$ Dept of Respiratory Medicine,

Heartlands Hospital Birmingham, and \#Host Defence Unit, Royal Brompton Hospital, London, UK.

CORRESPONDENCE

R. Wilson

Royal Brompton Hospital

Sydney Street

London SW3 6NP

UK

Fax: 442073518338

E-mail: r.wilson@rbht.nhs.uk

Received:

November 242005

Accepted after revision:

March 272006 
have been reported previously. Aspergillomas may originate in large cavity lesions of inactive M. kansasii infection [13] and may complicate pulmonary disease caused by M. xenopi [14]. Patients with $M$. malmoense or $M$. avium intracellulare complex (MAC) infection may have concomitant CNPA [9]. There is also potential for confusion in the diagnosis of NTM infection and Aspergillus-related lung disease, as both entities can have radiological features of tree-in-bud, nodules and cavities.

In patients with bronchiectasis, it is not clear whether NTM infection predisposes Aspergillus-related lung disease. This prompted the present authors to examine whether, in cases of bronchiectasis, serological markers and radiological features for Aspergillus-related lung disease are positive more often in patients with NTM disease compared to those without.

\section{METHODS}

A case-control study was conducted that included consecutive patients with bronchiectasis and NTM seen in the Host Defence Unit (Royal Brompton Hospital, London, UK) during 1995-2003. Cases with NTM were identified through sputum examination performed as part of routine screening due to suggestive symptoms or radiology results suggestive of NTM disease. Patients with bronchiectasis are checked for NTM disease annually; if symptoms or radiology change, patients are tested more frequently.

Sputum samples were processed for mycobacteria using the modified Petroff's 4\% sodium hydroxide method [15]. The processed samples were then inoculated onto two LowensteinJensen slopes (one with pyruvate and one with glycerol). Acid fast bacilli-positive smear samples were also inoculated into Middlebrook 7H12 Bactec vials (Becton Dickinson and Co., Sparks, Maryland, MD, USA). Positive cultures were identified via biochemical and temperature tests and, where appropriate, Accuprobe (Gen-Probe Inc., San Diego, CA, USA) [16]. A group of newly referred consecutive patients assessed in the Host Defence Unit who underwent a planned protocol of investigations of their conditions and who did not have evidence of NTM served as controls. Cases with NTM were culture positive on three or more occasions for a single NTM species, whereas controls were culture negative.

Patients' characteristics, the underlying cause of bronchiectasis, the presence of peripheral blood eosinophilia and the severity of bronchiectasis using lung function tests, including forced expiratory volume in one second (FEV1), were recorded. Aspergillus serology data, including A. fumigatus radioallergosorbent test (RAST), total serum immunoglobulin (Ig)E and Aspergillus precipitins were collected and sputum was examined for presence of A. fumigatus. Positive Aspergillus serology was classified as one or more positive result of either Aspergillus RAST and/or Aspergillus precipitins. The cut-off for Aspergillus RAST was $10 \mathrm{IU} \cdot \mathrm{L}^{-1}$. Aspergillus RAST and precipitins are specific tests for $A$. fumigatus, the most important species for lung disease.

High-resolution computed tomography (HRCT) scans were examined for evidence of aspergilloma, consisting of an upper lobe, mobile intra-cavitary mass with an air crescent in the periphery [17]. Since the radiological features of ABPA, including central bronchiectasis and pulmonary infiltrates, are difficult to differentiate in patients with bronchiectasis and
NTM infection, clinical and serological criteria, including episodic bronchial obstruction (asthma), peripheral blood eosinophilia and positive Aspergillus serology, were used to diagnose ABPA [18]. HRCT scans were evaluated for features of CNPA: multiple nodules, focal consolidation, tree-in-bud appearance and mycetoma formation [7].

For statistical analysis, characteristics and findings of patients with NTM were compared with those without, initially in a univariate analysis using Chi-squared or Fisher's exact test for contingency tables and unpaired t-tests for means. A multivariate logistic regression model was constructed to examine the association between NTM and Aspergillus-related lung disease with adjustment for confounding effects of other variables, such as underlying lung function. This enabled estimation of the association using odds ratios (ORs) and confidence intervals (CIs).

\section{RESULTS}

A total of 34 cases had bronchiectasis and NTM; four of these had missing data. The NTM species isolated were $M$. avium intracellulare in 18 patients, $M$. kansasii in five patients, $M$. chelonae in two patients, M. malmoense in two patients, $M$. fortuitum in two patients and M. simii in one patient. All patients met the American Thoracic Society criteria for NTM disease [19] with more than three positive cultures. Of the 30 patients with NTM, 10 had radiological evidence of cavities, whereas none of the controls had cavitary lesions.

The characteristics of the patients in the univariate analyses are shown in table 1 . Cases with NTM were characterised by older age $(p=0.002)$ and lower FEV1 levels $(p=0.02)$. The predominant aetiologies of underlying bronchiectasis were different in the two groups. Moreover, lung function was worse in both cases and controls with positive Aspergillus markers: mean FEV1 was $1.30 \mathrm{~L}$ in cases with positive Aspergillus markers and $1.82 \mathrm{~L}$ in those without $(\mathrm{p}=0.09)$, whereas in controls with positive markers, FEV1 was $1.47 \mathrm{~L}$ and among those without it was $2.07 \mathrm{~L}(\mathrm{p}=0.08)$. Overall, the difference in FEV1 between those with and without Aspergillus markers was significant $(p=0.01)$ in a multiple linear regression analysis controlling for NTM disease status.

As seen in table 2, 10 out of 30 patients had positive serological markers for Aspergillus and six had the radiological features of Aspergillus-related lung disease. All 10 patients with positive serology had positive Aspergillus precipitins, three had positive Aspergillus RAST and four also had positive sputum. One patient with an aspergilloma also had A. fumigatus in sputum. Among 61 controls, there were six patients with positive serology, of which only one had positive precipitins and RAST. A. fumigatus in sputum was isolated from one patient without serological or radiological features of Aspergillus-related lung disease. There was no radiological evidence of CNPA or aspergilloma among control patients.

Positive Aspergillus serology was more prevalent in cases with NTM disease than in controls (10 out of 30 versus six out of 61 ; $\mathrm{p}=0.005)$, and this finding persisted after adjustment for FEV1 levels (adjusted OR 3.6, 95\% CI 1.1-11.6; $p=0.03$ ). The radiological features of Aspergillus-related lung disease were also more frequent in patients with NTM disease (six out of 30 versus none out of $61 ; \mathrm{p}=0.003)$. As shown in table 3 , the 


\begin{tabular}{|c|c|c|c|c|}
\hline TABLE 1 & \multicolumn{4}{|c|}{$\begin{array}{l}\text { Characteristics of patients with bronchiectasis } \\
\text { according to nontuberculous mycobacterial } \\
\text { (NTM) lung disease }\end{array}$} \\
\hline \multicolumn{2}{|c|}{ Characteristics } & With NTM ${ }^{\#}$ & Without NTM" & p-value ${ }^{+}$ \\
\hline \multicolumn{2}{|c|}{ Mean age yrs } & $62.2 \pm 14.6$ & $51.6 \pm 15.3$ & 0.002 \\
\hline \multicolumn{5}{|l|}{ Sex } \\
\hline \multicolumn{2}{|l|}{ Male } & 9 & 23 & 0.46 \\
\hline \multicolumn{2}{|l|}{ Female } & 21 & 38 & \\
\hline \multicolumn{5}{|c|}{$\begin{array}{l}\text { Underlying aetiology of } \\
\text { bronchiectasis }^{5}\end{array}$} \\
\hline \multicolumn{2}{|l|}{ Idiopathic } & 10 & 27 & 0.002 \\
\hline \multicolumn{2}{|c|}{ Post-tuberculosis } & 4 & 0 & \\
\hline \multicolumn{2}{|c|}{ Post-adult infection } & 1 & 10 & \\
\hline \multicolumn{2}{|c|}{ Post-childhood infection } & 2 & 8 & \\
\hline \multicolumn{2}{|c|}{ Rheumatoid arthritis } & 2 & 1 & \\
\hline \multicolumn{2}{|c|}{ Primary ciliary dyskinesia } & 3 & 7 & \\
\hline \multicolumn{2}{|c|}{ Diffuse pan-bronchiolitis } & 0 & 2 & \\
\hline $\begin{array}{l}\text { Allergic bro } \\
\text { Aspergillo }\end{array}$ & \multicolumn{3}{|c|}{ Aspergillosis } & \\
\hline \multicolumn{2}{|c|}{ Yellow nail syndrome } & 0 & 3 & \\
\hline \multicolumn{2}{|c|}{ Miscellaneous } & 4 & 0 & \\
\hline \multicolumn{5}{|c|}{ Pulmonary function tests } \\
\hline \multicolumn{2}{|l|}{ FEV 1 L } & $1.6 \pm 0.8$ & $2.0 \pm 0.8$ & 0.02 \\
\hline \multicolumn{2}{|l|}{ RV \% pred } & $147 \pm 35$ & $152 \pm 45$ & 0.62 \\
\hline \multicolumn{2}{|c|}{$T L, C O \%$ pred } & $67 \pm 25$ & $77 \pm 15$ & 0.05 \\
\hline
\end{tabular}

Data are presented as mean \pm SD or $\mathrm{n}$. FEV 1 : forced expiratory volume in one second; RV: residual volume; \% pred: \% predicted; $T L, C O$ : transfer factor of the lung for carbon monoxide. ${ }^{\#}: \mathrm{n}=30 ;{ }^{\bullet}: \mathrm{n}=61 ;^{+}$: univariate analysis using Chisquared or Fisher's exact test for contingency tables and unpaired t-test for comparison of means; ${ }^{s}$ : the most predominant aetiology reported; some patients had two aetiologies.

association of positive Aspergillus serology and radiology was more prevalent in NTM disease than in controls (OR 7.01, 95\% CI 2.3-21.1; $\mathrm{p}=0.0005)$, and this finding persisted after adjustment for age and lung function (adjusted OR 5.1, 95\% CI 1.5-17.0; $\mathrm{p}=0.008)$.

\section{DISCUSSION}

This case-control study showed that positive Aspergillus serology and radiological features of Aspergillus-related lung disease were more common in bronchiectasis with NTM compared with controls. The observed association of NTM and positive Aspergillus markers was moderately strong, with the odds of positive Aspergillus serology being five times higher in cases with NTM exposure. There are several possible explanations as to why the association exists. Patients with NTM receive multiple broad-spectrum antibiotics before the diagnosis is made and this drives culture findings to fungal rather than bacterial infection. There is a common milieu factor favouring the organisms just as organisms are associated in CF [20]. A common host defence abnormality, steroid therapy or immunosuppression due to chronic disease may predispose to both NTM and Aspergillus-related lung disease. There may be a severity association and as FEV1 is an imperfect measure of disease severity, Aspergillus colonisation becomes a surrogate for disease severity associated with NTM. The lung disease of NTM is unusually destructive and causes cavities, independent of the level of airflow obstruction; this provides a favourable environment for $A$. fumigatus.

The validity of the present findings depends on the strength of the study's methods. Case-control design falls below cohort studies in the hierarchy of epidemiological evidence. However, it is the most suitable approach for rare diseases and it is a significant improvement over case series without control groups. The features of good case-control studies are minimisation of bias and confounding. The present authors verified case and control status using robust tests for diagnosis, thereby reducing bias due to the risk of misclassification as much as possible. The key deficiency of the present study is its inability to confirm the diagnosis of Aspergillus-related lung disease with confidence due to its ubiquitous nature, a problem that is inherent in all such studies. The present case-control design is best suited to deal with this issue as the diagnostic errors are likely to affect both groups studied. Moreover, the present authors' multivariate analytical approach aimed to adjust for confounding factors that could lead to diagnostic imbalances between groups.

Further support for the present analytical approach comes from the clinical imperative to investigate patients with available tests, despite their deficiencies. ABPA is usually diagnosed using a variety of clinical, serological and radiological criteria [18]. With regard to radiological criteria, pulmonary infiltrates and central bronchiectasis are usually suggestive of ABPA. However, they may be encountered in severe bronchiectasis, such as $\mathrm{CF}$, as a result of chronic bacterial infection and might therefore be inappropriate as selective criteria for diagnosis [21]. NTM infection, especially MAC infection, can give rise to bronchiectasis and pulmonary infiltrates, as seen in ABPA $[10,11,22]$. In view of this, the present authors did not attempt to review CT scans for evidence of ABPA but relied on combined clinical and serological criteria as recommended for patients with $\mathrm{CF}$ [21]. Depending on severity of ABPA the serum IgE and IgG antibodies to A. fumigatus may be low or high [17]. Furthermore, patients with mild ABPA may have abnormal serological markers without suggestive CT features [23]. ABPA may be associated with an increased risk of invasive aspergillosis, especially when treated with corticosteroids [24]. In the present study, cases with NTM compared to controls had an increased rate of clinical and serological evidence of ABPA; however, this was not statistically significant.

Most aspergillomas develop in pre-existing tuberculous cavities, which frequently occur in the upper lobes and superior segment of the lower lobes. However, all cavities in the present study were found in the upper lobes. Lung cavities often become colonised with A. fumigatus, but it is their infrequent progression to aspergilloma formation or to invasive aspergillosis that leads to significant morbidity and mortality. The diagnosis of an aspergilloma is usually made from radiographical features, when an upper-lobe, mobile intra-cavitary mass with an air crescent in the periphery is present [17]. Patients usually have positive Aspergillus precipitins; however, some patients may be seronegative [18]. 


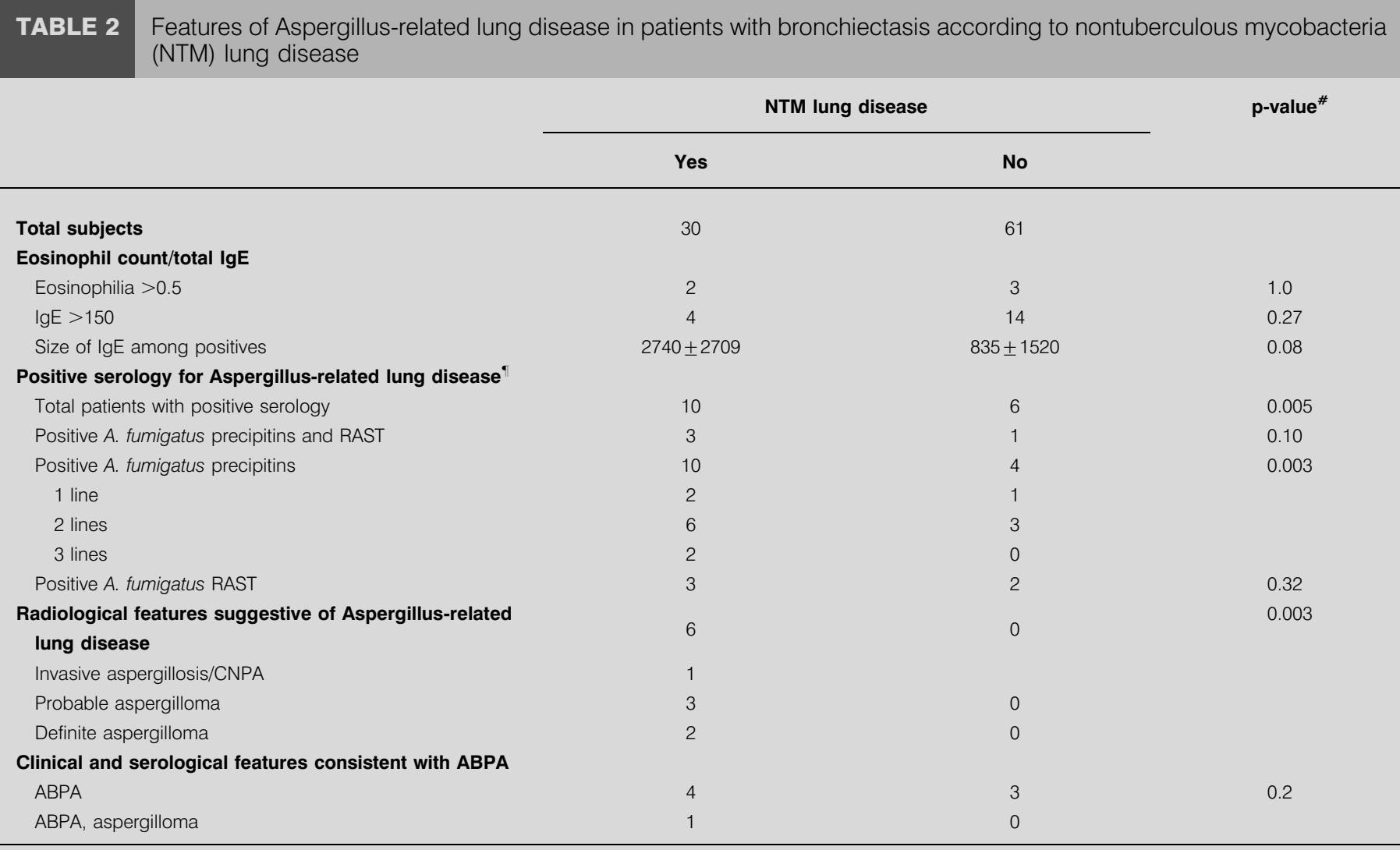

Data are presented as $\mathrm{n}$ or mean $\pm \mathrm{SD}$. Ig: immunoglobulin; A. fumigatus: Aspergillus fumigatus; RAST: radioallergosorbent test; CNPA: chronic necrotising pulmonary aspergillosis; ABPA: allergic bronchopulmonary aspergillosis. *: univariate analysis using Chi-squared or Fisher's exact test for contingency tables and unpaired t-test for comparison of means; ${ }^{\bullet}$ : see Methods section for details.

Although aspergillomas are often thought to be due to colonisations of the lung with $A$. fumigatus, the manifestations of Aspergillus-related lung disease overlap with a continuum between colonisation and tissue invasion. Invasive pulmonary aspergillosis may develop from an aspergilloma. In the present study, definite features of an aspergilloma occurred in two

\begin{tabular}{|c|c|c|c|c|c|}
\hline TABLE 3 & \multicolumn{5}{|c|}{$\begin{array}{l}\text { Logistic regression estimates of the association } \\
\text { between nontuberculous mycobacteria (NTM) } \\
\text { lung disease and Aspergillus-related lung } \\
\text { disease }\end{array}$} \\
\hline \multirow{2}{*}{\multicolumn{2}{|c|}{ Independent variable }} & \multicolumn{2}{|c|}{ Simple regression } & \multicolumn{2}{|c|}{ Multiple regression ${ }^{\#}$} \\
\hline & & OR $(95 \% \mathrm{Cl})$ & $\mathrm{p}$-value & OR $(95 \% \mathrm{Cl})$ & $\mathrm{p}$-value \\
\hline \multicolumn{2}{|c|}{$\begin{array}{l}\text { NTM lung disease } \\
\text { yes/no }\end{array}$} & $7.01(2.3-21.1)$ & 0.0005 & $5.1(1.5-17.0)$ & 0.008 \\
\hline \multicolumn{2}{|l|}{ FEV $_{1} \mathrm{~L}$} & $0.25(0.10-0.64)$ & 0.003 & $0.34(0.13-0.89)$ & 0.028 \\
\hline
\end{tabular}

OR: odds ratio; $\mathrm{Cl}$ : confidence interval; FEV1: forced expiratory volume in one second. \#: multiple logistic regression model with Aspergillus-related lung disease (serology and radiology combined) as the binary dependent variable and NTM lung disease, age and FEV1 as independent variables. Age did not contribute significantly to the model. See the Methods section for details. cases and probable features in three patients. Of these, one case had clinical and serological evidence of ABPA.

In invasive aspergillosis the use of antibodies against $A$. fumigatus has produced conflicting results, as patients in highrisk groups are frequently falsely seronegative. With antibody or antigen testing, serial assays appear more valuable than isolated tests, but specific recommendations about frequency of testing have not been established [25]. When faced with a positive respiratory specimen for $A$. fumigatus or positive Aspergillus precipitins the patient may be colonised or infected [18]. In immunosupressed patients e.g. post-transplantation, isolation of $A$. fumigatus from a respiratory specimen is considered to have a high positive predictive value of invasive aspergillosis [5]. In immune-competent patients with CNPA, however, reliable diagnostic criteria are not available and histopathological confirmation cannot always be obtained $[6,8]$.

Sputum examination often gives false-positive results in Aspergillus-related lung disease; the present study therefore did not include an isolated positive $A$. fumigatus in sputum without positive radiology or serology in the analysis. It is not clear what positive predictive value should be attributed to positive Aspergillus serology in patients with bronchiectasis and NTM. One third of the present cases with NTM have large cavities, which might be colonised with $A$. fumigatus and give 
positive results of Aspergillus precipitins. In addition, radiographical findings in Aspergillus-related lung disease are not diagnostic, because other infections and conditions may sometimes produce similar pictures [26]. There is no gold standard for the diagnosis of ABPA and established diagnostic criteria can often only be partially fulfilled in certain patient groups [27]. In view of this difficulty and as the gold standard to ascertain a diagnosis of CNPA and aspergilloma is based on open lung biopsy or post mortem examination, it is impractical to undertake test accuracy studies. This issue is best addressed by observational studies of the type reported here.

Combined serology, sputum and radiology for Aspergillusrelated lung disease will help reduce diagnostic uncertainty and inadequate treatment of NTM disease until new, more accurate tests become commonly available. There are many examples of poor outcome in NTM disease and coexisting Aspergillus-related lung disease: a case series of four patients with NTM infection who deteriorated or relapsed after treatment due to concomitant Aspergillus infection has been reported [9]. The radiological appearances of these patients were in keeping with CNPA. Other recent case reports have identified concomitant infection with Aspergillus as a possible reason for failure to respond to antimycobacterial chemotherapy. BOLLERT et al. [3] reported co-infection by M. malmoense and Aspergillus in three patients, all of whom died despite antimycobacterial and antifungal treatment. Two of the three patients showed evidence of an aspergilloma at post mortem examination. Similarly, DeBIEUVRE [28] reported a fatal case of M. malmoense complicated by co-infection with Aspergillus. Two other case series with poor outcome have described complex aspergillomas complicating M. kansasii and M. xenopi infections [13, 14].

The present authors propose that there is an association between nontuberculous mycobacteria and Aspergillus-related lung disease, which is important for diagnosis of the disease in clinical practice. Whether or not there is a causal relationship needs to be determined by further studies. It would also be useful to know if routine screening and early treatment of Aspergillus-related lung disease leads to an improvement in outcome compared with standard care. Due to rarity of the condition, it would be extremely difficult to launch a randomised trial, so more robust observational studies are required. The practical implication of the present findings is that in patients with bronchiectasis and nontuberculous mycobacteria infection it is important to investigate for coexisting Aspergillus-related lung disease infection early, at the time of starting treatment for nontuberculous mycobacteria. This is because the outcome of such cases is generally poor $[3,28]$ and treatment of the fungal disease might improve the outcome. If initially not diagnosed, Aspergillus-related lung disease may be subsequently mistaken as treatment failure or as relapse of nontuberculous mycobacteria infection [9].

\section{REFERENCES}

1 Management of opportunist mycobacterial infections, Joint Tuberculosis Committee Guidelines 1999. Subcommittee of the Joint Tuberculosis Committee of the British Thoracic Society. Thorax 2000; 55: 210-218.
2 Marras TK, Daley CL. Epidemiology of human pulmonary infection with nontuberculous mycobacteria. Clin Chest Med 2002; 23: 553-567.

3 Bollert FG, Sime PJ, MacNee W, Crompton GK. Pulmonary Mycobacterium malmoense and aspergillus infection: a fatal combination? Thorax 1994; 49: 521-522.

4 Mussaffi H, Rivlin J, Shalit I, Ephros M, Blau H. Nontuberculous mycobacteria in cystic fibrosis associated with allergic bronchopulmonary aspergillosis and steroid therapy. Eur Respir J 2005; 25: 324-328.

5 Munoz P, Alcala L, Sanchez CM, et al. The isolation of Aspergillus fumigatus from respiratory tract specimens in heart transplant recipients is highly predictive of invasive aspergillosis. Transplantation 2003; 75: 326-329.

6 Saraceno JL, Phelps DT, Ferro TJ, Futerfas R, Schwartz DB. Chronic necrotizing pulmonary aspergillosis: approach to management. Chest 1997; 112: 541-548.

7 Buckingham SJ, Hansell DM. Aspergillus in the lung: diverse and coincident forms. Eur Radiol 2003; 13: 1786-1800.

8 Gefter WB, Weingrad TR, Epstein DM, Ochs RH, Miller WT. "Semi-invasive" pulmonary aspergillosis: a new look at the spectrum of aspergillus infections of the lung. Radiology 1981; 140: 313-321.

9 Hafeez I, Muers MF, Murphy SA, Evans EG, Barton RC, McWhinney P. Non-tuberculous mycobacterial lung infection complicated by chronic necrotising pulmonary aspergillosis. Thorax 2000; 55: 717-719.

10 Ellis SM, Hansell DM. Imaging of non-tuberculous (atypical) mycobacterial pulmonary infection. Clin Radiol 2002; 57: 661-669.

11 Hollings NP, Wells AU, Wilson R, Hansell DM. Comparative appearances of non-tuberculous mycobacteria species: a CT study. Eur Radiol 2002; 12: 2211-2217.

12 Jeong YJ, Lee KS, Koh WJ, Han J, Kim TS, Kwon OJ. Nontuberculous mycobacterial pulmonary infection in immunocompetent patients: comparison of thin-section CT and histopathologic findings. Radiology 2004; 231: 880-886.

13 Maliwan N, Zvetina JR. Pulmonary mycetoma following Mycobacterium kansasii infection. Report of seven cases. Arch Intern Med 1985; 145: 2180-2183.

14 Johnston ID. Mycobacterium xenopi infection and aspergilloma. Tubercle 1988; 69: 139-143.

15 Collins CH, Lyne PM, Grange JM, et al. Microbiological Methods. 8th Edn. London, Hodder Arnold, 2004; pp. 60-178.

16 American Society of Microbiology. Manual of Clinical Microbiology. 7th Edn. Washington, American Society of Microbiology, 1999.

17 Soubani AO, Chandrasekar PH. The clinical spectrum of pulmonary aspergillosis. Chest 2002; 121: 1988-1999.

18 Stevens DA, Kan VL, Judson MA, et al. Practice guidelines for diseases caused by Aspergillus. Infectious Diseases Society of America. Clin Infect Dis 2000; 30: 696-709.

19 Diagnosis and treatment of disease caused by nontuberculous mycobacteria, This official statement of the American Thoracic Society was approved by the Board of Directors, March 1997. Medical Section of the American Lung Association. Am J Respir Crit Care Med 1997; 156: S1-S25.

20 Olivier KN, Weber DJ, Lee JH, et al. Nontuberculous mycobacteria. II: nested-cohort study of impact on cystic fibrosis lung disease. Am J Respir Crit Care Med 2003; 167: 835-840. 
21 Mastella G, Rainisio M, Harms HK, et al. Allergic bronchopulmonary aspergillosis in cystic fibrosis. A European epidemiological study. Epidemiologic Registry of Cystic Fibrosis. Eur Respir J 2000; 16: 464-471.

22 Hartman TE, Swensen SJ, Williams DE. Mycobacterium avium-intracellulare complex: evaluation with CT. Radiology 1993; 187: 23-26.

23 Kumar R. Mild, moderate, and severe forms of allergic bronchopulmonary aspergillosis: a clinical and serologic evaluation. Chest 2003; 124: 890-892.

24 Ganassini A, Cazzadori A. Invasive pulmonary aspergillosis complicating allergic bronchopulmonary aspergillosis. Respir Med 1995; 89: 143-145.
25 Tomee JF, Mannes GP, van der BW, et al. Serodiagnosis and monitoring of Aspergillus infections after lung transplantation. Ann Intern Med 1996; 125: 197-201.

26 Kuhlman JE, Fishman EK, Siegelman SS. Invasive pulmonary aspergillosis in acute leukemia: characteristic findings on CT, the CT halo sign, and the role of CT in early diagnosis. Radiology 1985; 157: 611-614.

27 Taccetti G, Procopio E, Marianelli L, Campana S. Allergic bronchopulmonary aspergillosis in Italian cystic fibrosis patients: prevalence and percentage of positive tests in the employed diagnostic criteria. Eur J Epidemiol 2000;16: 837-842.

28 Debieuvre D. Pulmonary Mycobacterium malmoense and Aspergillus infection. Thorax 1995; 50: 216. 